\title{
Modélisation de la houle cyclonique dans le lagon de Mayotte
}

\author{
Ywenn DE LA TORRE ${ }^{1}$, Sophie LECACHEUX ${ }^{2}$, Rodrigo PEDREROS ${ }^{2}$ \\ et Yann BALOUIN ${ }^{3}$ \\ ${ }^{1}$ BRGM, SGR La Réunion, 5 rue Ste Anne BP906, 97478 St Denis Cedex \\ y.delatorre@brgm.fr \\ ${ }^{2}$ BRGM, ARN, 3 av. C. Guillemin, BP6009, 45060 Orléans Cedex 2 \\ s.lecacheux@brgm.fr \\ r.pedreros@,brgm.fr
}

${ }^{3}$ BRGM, SGR Languedoc Roussillon, 1039 rue de Pinville, 34000 Montpellier y.balouin@,brgm.fr

\section{Résumé :}

L'île de Mayotte est exposée aux submersions marines liées aux surcotes d'origine cyclonique. Or, le rôle de la houle cyclonique sur ces surcotes reste aujourd'hui peu connu. L'objectif de ces travaux est d'estimer les surcotes liées à la houle cyclonique (set-up) afin de mieux appréhender leur influence sur les submersions marines. Pour cela le modèle SWAN a été mis en place et forcé par des vents cycloniques (SeaWinds, NOAA) et les conditions de vagues environnantes (modèle WaveWatch3, NOAA). Les simulations réalisées sur des scenarios réels et théoriques font apparaître des hauteurs de houle (Hs) relativement importantes (jusqu'à $7 \mathrm{~m}$ à l'ouest du lagon) tandis que le set-up reste globalement modéré (moins d'1 m), même dans les zones fermées du lagon.

\begin{abstract}
:
Mayotte Island is exposed to cyclonic surges but the wave impact on these surges is almost unknown. The aim of this work is to estimate the cyclonic wave set-up in order to understand its influence on surges. SWAN model was implemented and forced by cyclonic wind (SeaWinds), by waves (WaveWatch3) and by global data. Based on real and theoretical cyclones scenarios, simulations show a high exposition of wave height (up to $7 \mathrm{~m}$ in the west lagoon) whereas the set-up seems moderate (less than $1 \mathrm{~m}$ ) even in the closed lagoon areas.
\end{abstract}

\section{Mots-clés :}

Cyclone - Houle - Lagon - Mayotte - Modélisation - Surcote 


\section{$1 \quad$ Introduction}

Dans le cadre de la réalisation par le BRGM des atlas des aléas naturels à Mayotte (AUDRU et al., 2004), les menaces de submersion marine liées aux surcotes cycloniques ont été mises en évidence. Or, le rôle de la houle sur ces surcotes reste aujourd'hui mal connu à Mayotte.

Cette étude s'attache à la modélisation de la houle cyclonique à Mayotte afin de pouvoir disposer de valeurs de surcote liée à la houle (set-up) pour estimer leur influence sur les submersions marines.

L'objectif est donc de modéliser, pour différents cas de cyclones, l'élévation du niveau de la mer liée à la houle (hauteur de vague et set-up) arrivant sur le récifbarrière et dans le lagon de Mayotte.

Après un bref rappel du contexte cyclonique de Mayotte, cet article présente la méthodologie mise en œuvre puis les simulations pour les différents cas de cyclones étudiés. Ces résultats sont enfin discutés.

\section{Contexte cyclonique de Mayotte et choix de scenarii}

L'île de Mayotte est localisée dans le bassin sud-ouest de l'Océan Indien. Elle est la plus orientale des quatre îles de l'archipel des Comores, archipel volcanique au Nord du canal du Mozambique, situé entre les côtes africaines et le Nord de Madagascar (figure 1).

Mayotte $\left(375 \mathrm{~km}^{2}\right)$ est composée d'une île principale, Grande Terre $\left(360 \mathrm{~km}^{2}\right)$, où réside $90 \%$ de la population, entourée de nombreux îlots éparses dont le plus important et le seul à être occupé de manière permanente est celui de Petite-Terre.

Cet ensemble est ceinturé au large par une barrière corallienne, ouverte par plusieurs passes, isolant un vaste lagon de $1000 \mathrm{~km}^{2}$ environ. L'ensemble des côtes mahoraises est protégée par ce récif, à l'exception de la façade Est de PetiteTerre exposée au large.

Le bassin cyclonique du Sud-Ouest de l'Océan Indien qui est suivi par le Centre des Cyclones Tropicaux de La Réunion connaît en moyenne une douzaine de systèmes dépressionnaires tropicaux par an. Mayotte bénéficie de la protection importante que constitue Madagascar et si l'on en juge par le détail des alertes, l'île n'a été concernée que par 10 cyclones depuis 1976 (plusieurs tempêtes tropicales ont également engendré des conséquences importantes).

Les trajectoires de cyclones passant à proximité de Mayotte ont un caractère très aléatoire (MAYOKA, 1998). Si globalement une majorité des évènements cycloniques semblent se diriger vers le sud-Ouest de Mayotte, ces systèmes peuvent aussi bien passer au Nord, au Sud, à l'Est qu'à l'Ouest de l'île. 

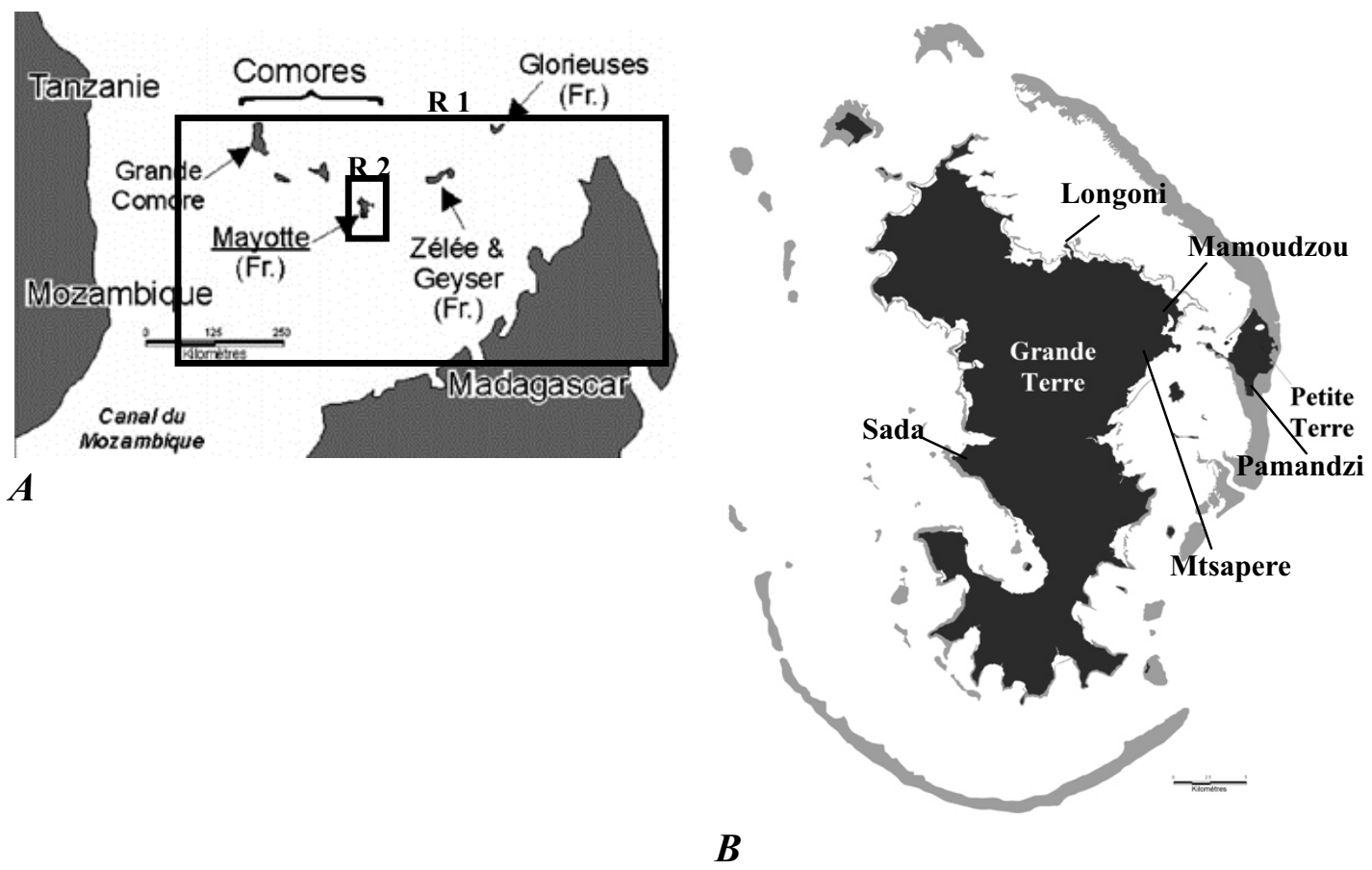

Figure 1. Localisation de Mayotte : emprise des grilles de calcul de Rang 1 et 2 (R1 et $R 2$ sur $A)$; toponymie citée dans l'article (B)

En raison du peu de cyclones observés sur la zone, nous avons eu recours à des cas théoriques pour représenter des situations probables mais non mesurées, en particuliers pour les cas extrêmes. En effet, la base de données du modèle de vague WaveWatch3 (NWW3) de la NOAA (National Oceanic and Atmospheric Administration, ftp://polar.ncep.noaa.gov/pub/history/waves/), fait apparaître que sur les dix dernières années de données disponibles, la houle de la tempête tropicale Gloria en 2000 est de loin la plus importante (plus de 6m). Or, il est connu localement que des cyclones plus anciens ont été plus impactant. Les valeurs de vent et de houle s'appuient donc sur des cas existants mais les trajectoires ont été modifiées afin de simuler différents niveaux d'exposition. Nous avons ainsi retenu 3 scenarios principaux :

1. «scenario modéré » basé sur le cas réel de Gloria (intensité max. au 2 mars 2000 à minuit soit $110 \mathrm{~km} / \mathrm{h}$ de vent moyen et $974 \mathrm{hPa}$ de pression au centre) qui suit une trajectoire NE-SW et passe à l'Est de Mayotte en restant au-dessus de Madagascar;

2. «scenarios intenses" basé sur les caractéristiques du cyclone intense Kamisy de 1984 (vent moyen de 120 km/h et pression à l'œil de $969 \mathrm{hPa}$ ) qui passe à environ $50 \mathrm{~km}$ de Mayotte, mais avec des trajectoires théoriques (N, NW, NE, E, W, SW, SE, S) ;

3. «scenario extrême», dans le cas théorique où Mayotte se situerait au 
niveau du mur de l'œil (rayon de vents maximums) pour un cyclone de l'intensité de Kamisy et des trajectoires SW et NE.

\section{$3 \quad$ Méthodologie}

\subsection{Modèle utilisé}

Le modèle SWAN (Simulating WAves Nearshore), développé à l'Université de Technologie de Delft (Pays-Bas), permet de calculer de façon réaliste les paramètres décrivant les vagues au niveau des zones côtières. Une description détaillée du modèle est faite dans BOOIJ et al. (2004). SWAN tient notamment en compte des phénomènes de dissipation liée au déferlement des vagues et au frottement au fond. Ces deux phénomènes étant particulièrement importants en présence de récifs (Gourlay, 1996a et b; Massel et Gourlay, 2000; Massel et Brinkman, 2001). Aussi SWAN a déjà été utilisé en présence de récifs artificiels (Muilwijk, 2005) et naturels (Stone et al. 2005 ; Lowe et al. 2008).

SWAN est capable en outre de calculer la surcote induite par la houle en résolvant les équations de tensions de radiations.

\subsection{Grilles de calcul}

Les simulations ont été réalisées sur la base d'une modélisation " emboitée ». Une grille de grande emprise allant du Nord de Madagascar aux Comores fournit les conditions aux limites à une grille de plus faible emprise centrée sur Mayotte et son lagon (figure 1).

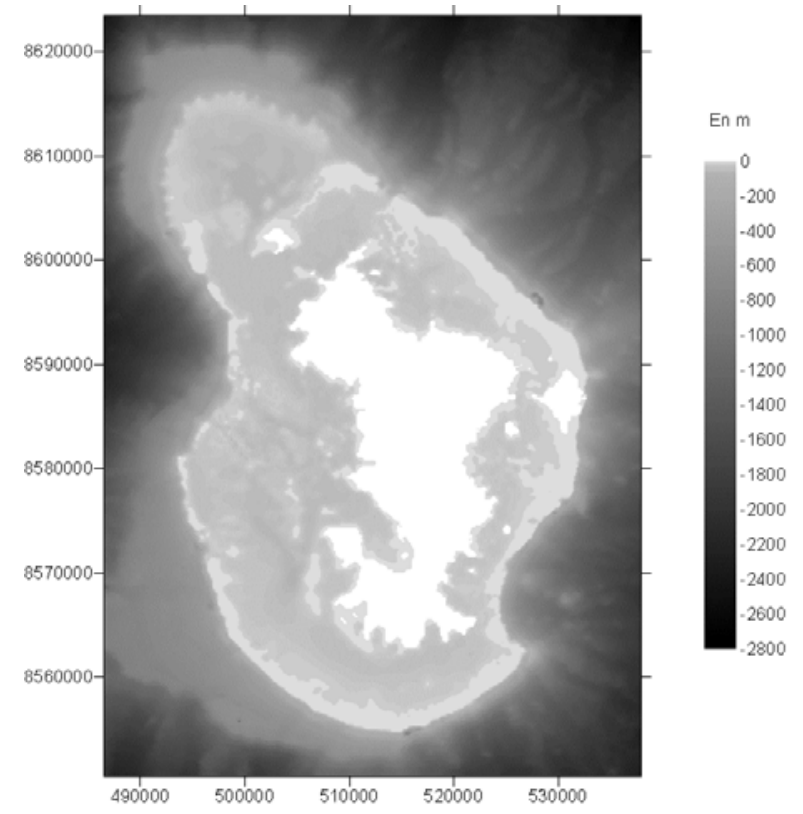

Figure 2. Grille de Rang 2 
La grille de grande emprise permet de réaliser les simulations de « Rang 1 ». Elle a été constituée à partir des données bathymétriques issues de la base de données GEBCO (General Bathymetric Chart of the Oceans). Les informations bathymétriques contenues dans GEBCO résultent d'une compilation à l'échelle du globe de sondages bathymétriques et de données issues de l'altimétrie satellitale. La taille de la maille est de 1 minute x 1 minute soit environ $1,85 \mathrm{~km} \times 1,85 \mathrm{~km}$.

La grille de plus faible emprise, dite de Rang 2 (figure 2), a été constituée à partir de la compilation de données du SHOM, complétées par des données de sondage multifaisceaux issues de la campagne BATHYMAY (AUDRU et al., 2006) sur la pente externe du récif barrière, et des données terrestres de l'IGN. La résolution de la grille de Rang 2 est de $250 \mathrm{~m}$.

\subsection{Données initiales}

En l'absence de données in situ sur la houle cyclonique à Mayotte, des méthodes d'initialisation différentes ont été appliquées à chaque cas (tableau 1).

Tableau 1. Scenarios et données initiales correspondantes

\begin{tabular}{|c|c|c|c|}
\hline \multicolumn{2}{|c|}{ SCENARII } & \multicolumn{2}{c|}{ Données d'entrée } \\
\hline CAS & Directions des vagues & VAGUES & VENT \\
\hline Réel modéré : GLORIA & ouest & NWW3 & SeaWinds \\
\hline $\begin{array}{c}\text { Théorique plus puissant }: \\
\text { type KAMISY }\end{array}$ & 8 directions & $\begin{array}{c}\text { Forçage } \\
\text { constant } \\
(6 \mathrm{~m})\end{array}$ & $\begin{array}{c}\text { Forçage } \\
\text { constant } \\
(32 \mathrm{~m} / \mathrm{s})\end{array}$ \\
\hline $\begin{array}{c}\text { Théorique extrême : type } \\
\text { KAMISY }\end{array}$ & SO et NE & $\begin{array}{c}\text { Forçage } \\
\text { constant } \\
(12 \mathrm{~m})\end{array}$ & $\begin{array}{c}\text { Forçage } \\
\text { constant } \\
(50 \mathrm{~m} / \mathrm{s})\end{array}$ \\
\hline
\end{tabular}

Ces données concernent :

- les résultats du modèle WaveWatch3 depuis 1997 (hauteur significative, période pic, et direction pic des vagues) ont été appliquées aux frontières du Rang 1 afin de tenir compte des conditions environnantes (les vagues n'étant pas générées par le cyclone);

- associés aux champs de vent SeaWinds issus de l'altimétrie satellitale depuis 1987 et suivant une grille de $0,25^{\circ} \times 0,25^{\circ}$ avec une fréquence de $6 \mathrm{~h}$, opéré également par la NOAA (http://www.ncdc.noaa.gov/oa/rsad/seawinds.html) ;

- et pour les événements antérieurs à 1990, un vent constant a été appliqué sur toute la grille de Rang 2. Celui-ci a été déterminé à partir de l'étude de METEO-FRANCE (2003) faisant appel à la reconstitution des champs de vent à partir de la trajectoire des cyclones, Les conditions limites de houle ont été 
calculées avec les abaques de l'USACE (US Army Corps of Engineers, 2001) et les paramètres des cyclones issus du même rapport de METEO-FRANCE.

Les marées astronomiques ont été extraites à partir du logiciel WXtide 4.7 pour chaque période considérée.

\section{$4 \quad$ Résultats des simulations}

Les simulations permettent de mettre en évidence les situations suivantes :

- pour le «scenario modéré » (tempête tropicale Gloria), l'impact reste relativement faible grâce à la protection que représente Madagascar (figure 4). Les houles ne dépassent pas $1.5 \mathrm{~m}$ (Hs) au large et le set-up est négligeable dans le lagon ;

- pour les «scenarios intenses" (cyclone de classe 5 de type Kamisy), les valeurs maximum de houle atteignent entre 4 et $6 \mathrm{~m}$ dans le lagon selon les directions (figure 5A) avec un set-up allant jusqu'à $25 \mathrm{~cm}$ en certains points ;

- enfin, « les scenarios extrêmes » (en faisant l'hypothèse que Mayotte est située au niveau du rayon de vent maximum pour une intensité de cyclone de type Kamisy), les vagues peuvent atteindre $7 \mathrm{~m}$ dans le lagon et le set-up $1 \mathrm{~m}$ sur les récifs (figure 5B).

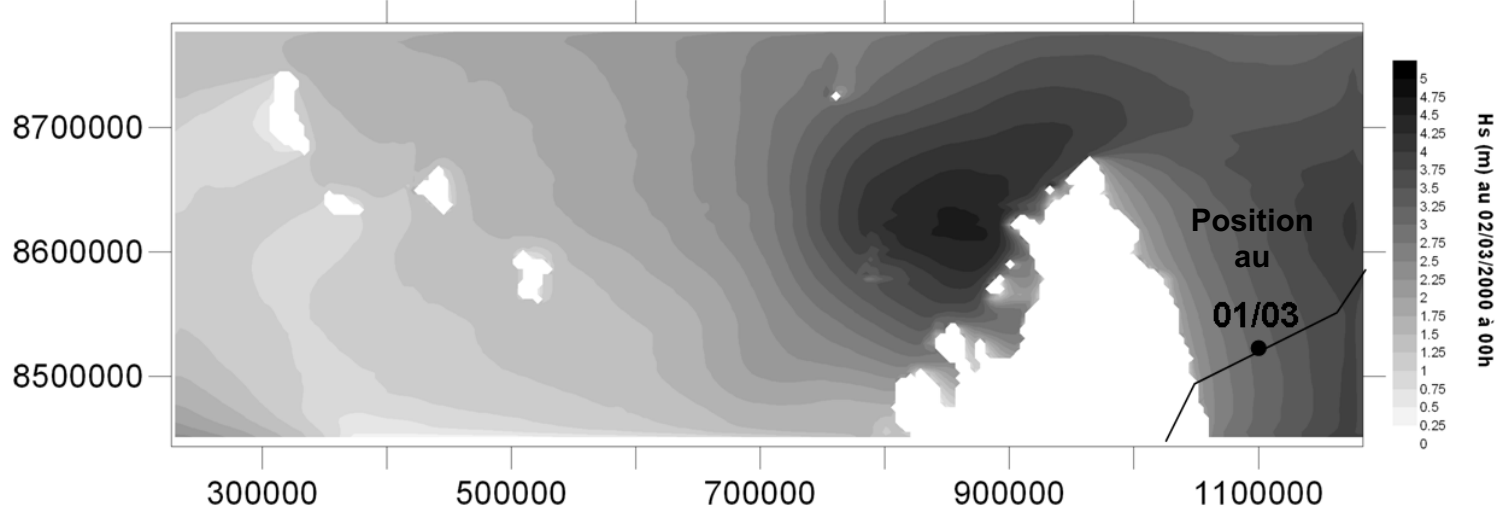

Figure 4.Protection offerte par Madagascar au Rang 1 pour le cas réel Gloria 


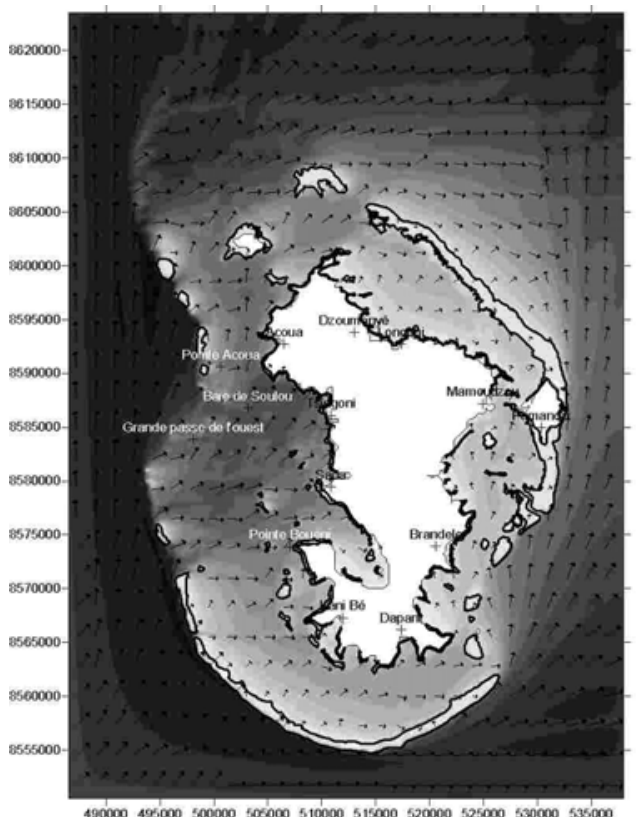

A
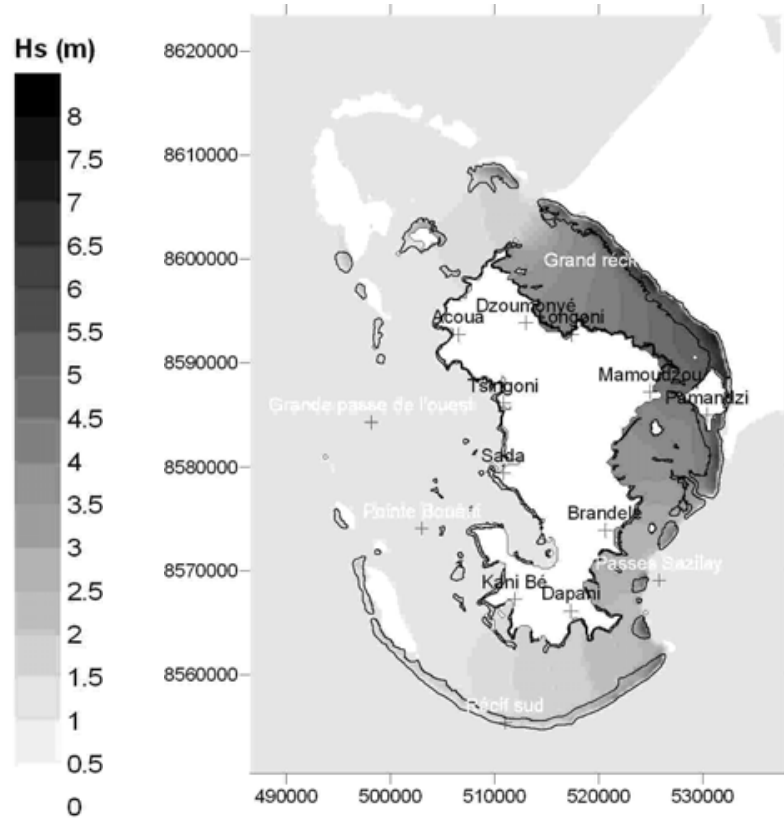

B

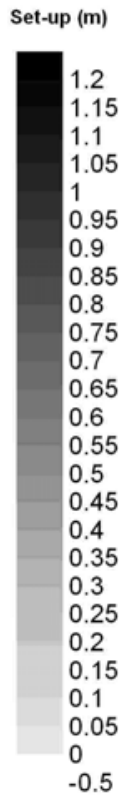

Figure 5. Exemples de simulations de Rang 2 (en $\mathrm{m}$ ) :

Hs pour une houle de sud-ouest du cyclone Kamisy (A) ; set-up pour une marée basse et une houle de nord-est d'un cyclone très intense potentiel (B)

La marée haute permet le passage de vagues plus hautes dans le lagon mais c'est à marée basse que le set-up est le plus sensible $(35 \mathrm{~cm}$ à marée haute pour $1 \mathrm{~m}$ à marée basse).

Pour le «scenario extrême », la côte ouest est la plus touchée par les vagues tandis que le set-up concerne davantage les secteurs sud et nord-est.

Sur quelques zones à enjeux de l'île (Longoni, Mamoudzou, Sada, M'Tsapéré figure 1), les résultats font apparaître un set-up modéré (entre 14 et $25 \mathrm{~cm}$ ) pour une exposition à la houle toutefois relativement importante $(4 \mathrm{~m}$ pour Sada figure 6 - et $3 \mathrm{~m}$ pour les autres sites cités) pour Mayotte. Quant à l'aéroport de Pamandzi, il est bien protégé des vagues (maximum $1 \mathrm{~m}$ ) et le set-up est maximal pour une houle d'est $(18 \mathrm{~cm})$. 

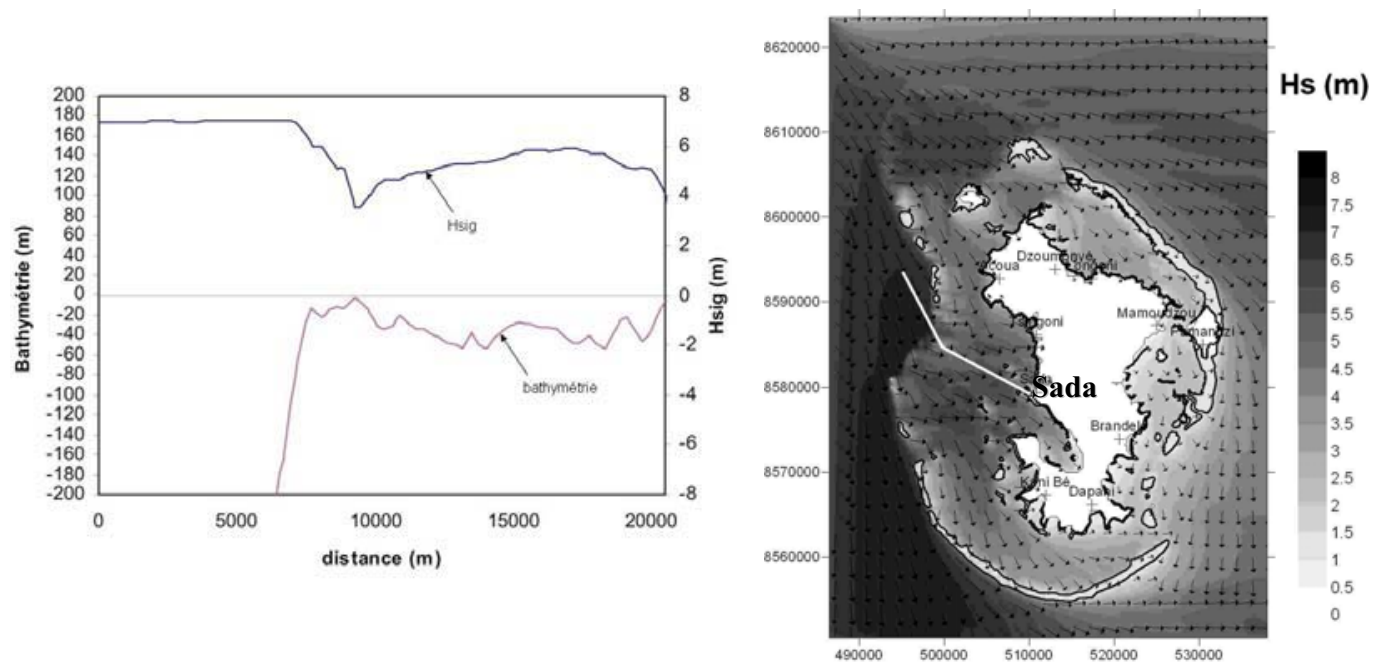

Figure 6. Section allant du large de Mayotte (ouest) à la ville de Sada pour un cyclone type Kamisy, la houle provient du nord-ouest

\section{$5 \quad$ Discussion des résultats}

\subsection{Influence du set-up et de la houle}

Les simulations réelles et théoriques d'événements cycloniques modérés à forts mettent en évidence une surcote liée à la houle relativement faible, quelque soit l'intensité de la houle cyclonique.

Ces résultats semblent contredire le principe selon lequel l'influence du set-up est prépondérant sur la surcote atmosphérique dans des environnements à fortes pentes et récifs tels que Mayotte (WEAVER, 2004). En effet, pour le cyclone Kamisy, les valeurs de set-up calculées sont inférieures aux données de surcote atmosphérique modélisées par METEO-FRANCE (2003).

De plus, les plus hautes valeurs de set-up (1m) sont observées à marée basse (l'amplitude de la marée est de $3 \mathrm{~m}$ en moyenne), ce qui contribue à attribuer au set-up un rôle limité en terme d'élévation totale du plan d'eau dans le lagon. Cependant, les valeurs de hauteur significative de la houle sont relativement élevées compte-tenu du caractère abrité de Mayotte $(6 \mathrm{~m}$ dans l'ouest du lagon pour le scenario " intense» et une houle de Nord-Ouest et pour le scenario « extrême » et une houle de Sud-Ouest).

Ainsi, en termes de submersions marines de la frange côtière, même si le rôle du set-up généré par le déferlement sur le récif barrière semble limité, les hauteurs de houle importantes dans le lagon laissent supposer un run-up (altitude maximale atteinte par le jet de rive) significatif sur les côtes potentiellement exposées. 


\subsection{Limites de l'approche numérique réalisée}

Tout d'abord, le manque de données d'entrée disponibles pour modéliser les différents cas de cyclones possibles nous a contraints à recourir à des cas de trajectoires théoriques (scenarios « intense » et « extrême ») et plus impactantes.

En outre, les données numériques du SHOM étant fragmentaires au moment des travaux, elles ont dues être complétées par des points de sonde issus de la carte de navigation, ce qui tend à sous-estimer les profondeurs du MNT (les cartes de navigation présentent les «points hauts » du fond afin de signaler les pinacles coralliens et autres haut-fonds);

Enfin, la contrainte majeure reste que l'absence de mesures de vagues sur Mayotte qui ne nous a pas permis de valider les différentes simulations effectuées.

\section{$6 \quad$ Conclusions et perspectives}

Ainsi, en raison de l'absence de validation possible à ce stade des travaux, ces résultats intermédiaires doivent être pris avec beaucoup de prudence et restent à améliorer. En l'état, les simulations montrent toutefois que la surcote liée à la houle resterait dans la plupart des cas relativement modérée (moins d'un mètre), même pour les zones fermées du lagon. Les hauteurs de houle pourraient être en revanche assez importantes dans l'Ouest du lagon (jusqu'à $6 \mathrm{~m}$ ), malgré le caractère «abrité » de Mayotte. Les zones sensibles de l'île sont relativement peu exposées en termes de hauteur de houle et de set-up, à l'exception de Sada où la houle pourrait atteindre $4 \mathrm{~m}$.

Afin de confirmer ces premiers résultats, des travaux complémentaires sont prévus, notamment en termes de bathymétrie (amélioration de la grille de Rang 2), de l'utilisation de champs de vent cyclonique pour les événements antérieurs à 1990 (modèle paramétrique basé sur la trajectoire du cyclone) et de calage/validation du modèle (réalisation de mesures in situ).

\section{$7 \quad$ Remerciements}

Ces travaux ont été financés par la Direction de l'Equipement de Mayotte et le BRGM.

\section{$8 \quad$ Références bibliographiques}

1 AUDRU JC, BITRI A., DESPRATS JF, MATHON C., MAURILLON SEDAN O., TERRIER-SEDAN M., ZORNETTE N (2004). Atlas des aléas naturels à Mayotte, Communes de Mamoudzou, Koungou, Dzaoudzi et Pamandzi. Rapport BRGM/RP-53037-FR, 135 p. 
2 AUDRU JC, GUENNOC P., THINON I., ABELARD O. (2006). Bathymay, la structure sous-marine de Mayotte révélée par l'imagerie multifaisceaux. C. R. Geoscience 338 (2006) 1240-1249, pp 1240-1249. 3 BATTJES J.A. et. JANSSEN J.P.F.M. (1978). Energy loss and set-up due to breaking of random waves, Proc. 16th Int. Conf. Coastal Engineering, ASCE, 569-587.

4 BOOIJ, N., HAAGSMA IJ.G.; HOLTHUIJSEN L.H.; KIEFTENBURG, A.T.M.M.; RIS, R.C.; VAN DER WESTHUYSEN A.J., ZIJLEMA M. (2004). Swan Cycle III version 40.41. User's Manual, 115p.

5 ELDEBERKY, Y., J.A. BATTJES. (1995). Parameterization of triad interactions in wave energy models, Proc. Coastal Dynamics Conf. '95, Gdansk, Poland, 140-148.

6 GOURLAY MR. (1996). Wave set-up on coral reefs 1. Set-up and wavegenerated flow on an idealized two dimensional horizontal reef. Coast Eng, 27, 161-193.

7 GOURLAY MR. (1996). Wave set-up on coral reefs 2 : Set-up on reefs with various profiles, Coast Eng, 28, 17-55.

8 LOWE R., FALTER J., MONISMITH S., ATKINSON M. (à paraître). Numerical modelling of a coastal reef-lagoon system. $11^{\text {th }}$ ICRS.

9 MASSEL S. R., BRINKMAN R.M. (2001). Wave-induced set-up and flow over shoals and coral reefs. Part 1. A simplified bottom geometry case. OCEANOLOGIA, 43 (4), pp. 373-388.

10 MASSEL S.R., GOURLAY M.R. (2000). On the modelling of wave breaking and set-up on coral reefs, Coast. Eng., 39, 1-27.

11 MAYOKA M. (1998). Les cyclones à La Réunion. Météo-France, Centre des Cyclones Tropicaux de La Réunion, $48 \mathrm{p}$.

12 METEO-FRANCE (2003). Surcotes liées au passage d'un cyclone à Mayotte. Rapport préliminaire destiné au BRGM. P. Daniel, Division Prévision Marine et Océanographie.

13 PHADKE, A.C., MARTINO C.D., CHEUNG K.F., HOUSTON S.H. (2003). Modeling of tropical cyclone winds and waves for emergency management. Ocean Engineering 30 pp.553-578.

14 STONE GW, ZHANG X, SHEREMET A. (2005). The role of barrier islands, muddy shelf and reefs in mitigating the wave field along coastal Louisiana. J. of Coastal Res. SI44 - pp 40-55.

15 U.S. ARMY CORPS OF ENGINEERS (2001). EM 1110-2-1100, Part II. Meteorology and Wave Climate.

16 WEAVER R.J. (2004). Effects of wave forces on storm surge. Master of Science thesis. Univ. of Florida, $65 \mathrm{p}$. 